\title{
Numerical Solution of Fractional-Order Fredholm Integrodifferential Equation in the Sense of Atangana-Baleanu Derivative
}

\author{
Jian Wang, ${ }^{1}$ Kamran $\mathbb{D}^{2},{ }^{2}$ Ayesha Jamal, ${ }^{2}$ and Xuemei Li $\mathbb{D}^{3}$ \\ ${ }^{1}$ Department of Basic Education and Research, Changchun Sci-Tech University, Changchun 130000, China \\ ${ }^{2}$ Department of Mathematics, Islamia College Peshawar, Khyber Pakhtoon Khwa, Peshawar, Pakistan \\ ${ }^{3}$ School of Architecture and Civil Engineering, Changchun Sci-Tech University, Changchun 130000, China
}

Correspondence should be addressed to Xuemei Li; xuemei1106aa@sina.com

Received 5 December 2020; Revised 30 December 2020; Accepted 2 January 2021; Published 12 February 2021

Academic Editor: Kamal Shah

Copyright (c) 2021 Jian Wang et al. This is an open access article distributed under the Creative Commons Attribution License, which permits unrestricted use, distribution, and reproduction in any medium, provided the original work is properly cited.

In the present article, our aim is to approximate the solution of Fredholm-type integrodifferential equation with Atangana-Baleanu fractional derivative in Caputo sense. For this, we propose a method based on Laplace transform and inverse LT. In our numerical scheme, the given equation is transformed to an algebraic equation by employing the Laplace transform. The reduced equation will be solved in complex plane. Finally, the solution of the given problem is obtained via inverse Laplace transform by representing it as a contour integral. Then, the trapezoidal rule is used to approximate the integral to high accuracy. We have considered linear and nonlinear fractional Fredholm integrodifferential equations to validate our method.

\section{Introduction}

Fractional calculus is the branch of mathematics which generalizes the concept of derivatives and integrals from integer to any positive real order [1-5]. Fractional-order derivatives are in fact definite integrals. For example, fractional derivative of a function geometrically accumulates the function. The corresponding accumulation includes the integer-order derivative as a special case. This shows that fractional calculus describes the global dynamics of realworld problems, whereas the classical calculus describes the local dynamics of the corresponding problems. Problems involving arbitrary order operators from engineering and other sciences are growing day by day. For example, applications of fractional calculus can be found in frequencydependent damping behavior of viscoelastic materials $[6,7]$, heat diffusion $[8,9]$, economics $[10]$, control theory [11], robotics, and other problems in engineering sciences, see [12] and references therein. Fractional-order operators can be used for accurate modeling of real-world problems [13]. In the literature, we can find a large amount of valuable work on fractional-order operators, see [14-19] and references therein. Also, a great effort has been made by the researchers for the existence and uniqueness of the analytical solutions of equations involving fractional operators, for example, see $[13,16,20]$.

However, in many cases, the analytical solutions of fractional differential equations cannot be obtained, so numerical methods must be used. In this regard, several numerical methods have been proposed for the approximation of differential or integrodifferential equations of arbitrary order. For example, the Legendre collocation method [21], sinc-collocation method [22], variational iteration method [23], Adomian decomposition method, see $[24,25]$ and references therein.

Many real-world phenomena [26-29] are analyzed via the solutions of fractional-order equations, which are derived from or based on Caputo-Liouville's operators $[15,19,30-32]$. Meanwhile, the study of fractional-order integrodifferential equations (FIDEs) is also much important as these equations occur in many phenomena of applied nature such as electromagnetic [33] and heat conduction 
[34]. Because of these interesting applications, FIDEs have gained great attention of the researchers [13, 35-38]. In this regard, numerous analytical and numerical methods have been developed for solving FIDEs. For example, in [15], the authors have approximated the FIDEs using the reproducing kernel Hilbert space method. In [35], the FIDEs are solved using the collocation technique. A collocation technique is presented in [39] for obtaining the approximate solution of FIDEs. Arikoglu and Ozkol [14] applied the differential transform method for the solution of FIDEs. A Legendre wavelet method [36] is proposed for the solution of FIDEs. FIDEs of Fredholm type are used in many applications in applied mathematics and physics and also for presenting physical time variation $[35,40]$. These can be used to resolve many phenomena in electrodynamics, fluid dynamics, and other fields of engineering and sciences [15].

In this article, the FIDEs of Fredholm type are considered with Atangana-Baleanu fractional derivative. In the literature, numerous definitions of arbitrary order derivatives are available, for example, the Caputo fractional derivative and Riemann-Liouville's fractional derivative [13, 16, 37, 41-47]. However, these derivatives contain singular kernel functions and they may face complications in modeling nonlocal real-world phenomena. In order to overcome these complications and model the nonlocal systems effectively, it is better to use Atangana-Baleanu (AB) derivative. In the literature, a large amount of work is available for the solution of differential or integrodifferential equations with $\mathrm{AB}$ derivative, see $[15,20,48-51]$ and references therein. In this article, we aim to approximate the solution of integrodifferential equations of Fredholm type with $\mathrm{AB}$ derivative of the form

$$
\begin{aligned}
{ }_{0}^{A B C} D_{t}^{\alpha} \mathcal{U}(t) & =\mathscr{H}(t, \mathcal{U}(t), \mathscr{T} \mathcal{U}(t)), \\
\mathcal{U}(0) & =\beta,
\end{aligned}
$$

where

$$
\begin{aligned}
& \mathscr{T} \mathscr{U}(t)=\int_{0}^{1} \mathscr{B}(t, \tau) \mathscr{Q}(\mathcal{U}(\tau)) \mathrm{d} \tau, \\
& \alpha \in(0,1], t, \quad \tau \in \mathscr{J}:[0,1], \beta \in \mathbb{R}, \mathscr{H}, \mathscr{B}, \mathscr{Q} \in \mathscr{C}[0,1],
\end{aligned}
$$

where ${ }_{0}^{A B C} D_{t}^{\alpha}$ is the Atangana-Baleanu Caputo derivative of order $\alpha$.

1.1. Preliminaries. Some basic definitions and lemma needed are as follows.

Definition 1. The left Riemann-Liouville (RL) integral for $\alpha>0$ is defined as [13]

$$
{ }_{0} I^{\alpha} \mathscr{U}(t)=\frac{1}{\Gamma(\alpha)} \int_{0}^{t} \frac{\mathcal{U}(s)}{(t-s)^{1-\alpha}} \mathrm{d} s .
$$

Definition 2. The right RL integral for $\alpha>0$ is defined as [13]

$$
{ }_{0} I^{\alpha} \mathscr{U}(t)=\frac{1}{\Gamma(\alpha)} \int_{t}^{0} \frac{\mathcal{U}(s)}{(s-t)^{1-\alpha}} \mathrm{d} s .
$$

Definition 3. The left RL derivative of order $0 \leq \alpha \leq 1$ is defined as [13]

$$
{ }_{0} D^{\alpha} \mathcal{U}(t)=\frac{d}{\mathrm{~d} t}\left(\frac{1}{\Gamma(1-\alpha)} \int_{0}^{t} \frac{\mathscr{U}(s)}{(t-s)^{\alpha}} \mathrm{d} s\right) .
$$

Definition 4. The right $\mathrm{RL}$ derivative of order $0 \leq \alpha \leq 1$ is defined as [13]

$$
{ }_{0} D^{\alpha} \mathcal{U}(t)=-\frac{d}{\mathrm{~d} t}\left(\frac{1}{\Gamma(1-\alpha)} \int_{0}^{t} \frac{\mathcal{U}(s)}{(t-s)^{\alpha}} \mathrm{d} s\right) .
$$

Definition 5. The Caputo derivative of fractional order $\alpha \in[0,1]$ is defined as [13]

$$
{ }_{0}^{c} D^{\alpha} \mathcal{U}(t)=-\frac{1}{\Gamma(1-\alpha)} \int_{0}^{t}(t-s)^{-\alpha} \mathcal{U}(s) \mathrm{d} s .
$$

Definition 6. The Mittag-Leffler function is defined as [13]

$$
\mathscr{E}_{\alpha}(t)=\sum_{m=0}^{\infty} \frac{t^{m}}{\Gamma(m \alpha+1)}, \quad \alpha>0,-\infty<t<\infty .
$$

Definition 7. The $A B$ derivative of order $\alpha$ is defined as [52]

$$
{ }_{0}^{A B C} D_{t}^{\alpha} \mathcal{U}(t)=\frac{M(\alpha)}{1-\alpha} \int_{0}^{t} \mathcal{U}^{\prime}(s) \mathscr{E}_{\alpha}\left[-\alpha \frac{(t-s)^{\alpha}}{1-\alpha}\right] \mathrm{d} s .
$$

Definition 8. The $A B$ fractional integral of order $\alpha$ is defined as [52]

$$
{ }_{0}^{A B C} I^{\alpha} \mathcal{U}(t)=\frac{1-\alpha}{M(\alpha)} \mathcal{U}(t)+\frac{\alpha}{M(\alpha)_{0}} I^{\alpha} \mathcal{U}(t) .
$$

The following hypothesis will be needed for the uniqueness and existence results of problems (1) and (2), which are based on fixed-point techniques [53]:

$A_{1}$ : suppose $\mathcal{U} \in \mathscr{C}[0,1]$ and $\mathscr{H} \in \Omega=(\mathscr{C}[0,1] \times \mathscr{J} \times$ $\mathscr{J} \times \mathscr{J}, \mathscr{J})$ is continuous function and there exist positive constants $\mathscr{L}_{1}, \mathscr{L}_{2}$, and $\mathscr{L}$ such that $\left\|\mathscr{H}\left(t, \mathscr{U}_{1}, \mathscr{V}_{1}, \mathscr{W}_{1}\right)-\mathscr{H}\left(t, \mathscr{U}_{2}, \mathscr{V}_{2}, \mathscr{W}_{2}\right)\right\| \leq \mathscr{L}_{1}\left(\| \mathscr{U}_{1}-\right.$ $\left.\mathscr{U}_{2}\|+\| \mathscr{V}_{1}-\mathscr{V}_{2}\|+\| \mathscr{W}_{1}-\mathscr{W}_{2} \|\right)$ for all $\mathscr{U}_{1}, \mathscr{V}_{1}, \mathscr{W}_{1} \mathscr{Y}$, $\mathscr{L}_{2}=\max _{t \in J}\|\mathscr{H}(t, 0,0,0)\|$, and $\mathscr{L}=\max \left\{\mathscr{L}_{1}, \mathscr{L}_{2}\right\}$. Let $\mathscr{X}$ be a Banach space and $\mathscr{Y}=\mathscr{C}[\mathscr{X}, \mathscr{J}]$ be the set of continuous functions defined on $\mathscr{J}$ with values in $\mathscr{X}$

$A_{2}$ : there exist positive constants $\mathscr{R}_{1}, \mathscr{R}_{2}$, and $\mathscr{R}$ such that $\quad\left\|\mathscr{T}_{1}\left(t_{1}, s_{1}, \mathscr{U}_{1}\right)-\mathscr{T}_{1}\left(t_{2}, s_{2}, \mathscr{U}_{1}\right)\right\| \leq \mathscr{R}_{1}\left(\| \mathscr{U}_{1}-\right.$ $\left.\mathscr{U}_{2} \|\right)$ for all $\mathscr{U}_{1}, \mathscr{U}_{2} \in \mathcal{Y}, \mathscr{R}_{2}=\max _{(t, \tau) \in D} \mathscr{T}_{1}(t, \tau, 0)$, and $\mathscr{R}=\max \left\{\mathscr{R}_{1}, \mathscr{R}_{2}\right\}$

$A_{3}$ : suppose $B_{r} \in\{\mathscr{U} \in \mathscr{Y}:\|\mathscr{U}\| \leq r\} \subseteq \mathscr{Y}$ for each $r>0$, defined as $r=\left(\|\beta\| /(1-p)^{-1}\right)$ and $p=(\mathscr{L}(\|\mathscr{U}\|+$ $\mathscr{R} t\|\mathcal{U}\|))$ 
$A_{4}$ : for each positive $r_{0}$, let $B_{r_{0}} \in\{\mathscr{U} \in(\mathscr{C}[0,1], \mathscr{J}):\|\mathscr{U}\| \leq r\}$, then clearly $B_{r_{0}}$ is closed, convex, and bounded subset in $(\mathscr{C}[0,1], \mathscr{J})$

Definition 9. $\mathscr{U} \in \mathscr{C}[0,1]$ is said to be the solution of (1) and(2), if $\mathscr{U}(0)=\beta$ and there exist $\mathscr{H} \in \Omega$ such that $t \in[0,1]$, then the equation

$$
\mathcal{U}(t)=\beta+_{0}^{A B} I^{\alpha} \mathscr{H}\left(t, \mathcal{U}(t), \mathscr{T}_{1} \mathcal{U}(t)\right),
$$

is satisfied.

Theorem 1. Let $\quad \mathcal{U} \in \mathscr{C}[0,1]$ such that ${ }_{0}^{A B C} D^{\alpha} \mathscr{U}(t) \in \mathscr{C}[0,1]$. Assume $A_{1}-A_{4}$ are satisfied, and if $\mathscr{H}\left(0, \mathcal{U}(0), \int_{0}^{1} \mathscr{B}(0, \tau) \mathscr{Q}(\mathcal{U}(\tau)) d \tau\right)=0 \quad$ and $(1-\alpha / M(\alpha))+\left(I^{\alpha} / M(\alpha) \Gamma(\alpha)\right) \leq 1$, then problems and(2) have a unique solution.

\section{The Laplace Transform Method for Fredholm Equations of Fractional Order}

In this section, we develop our numerical method for the approximation of the solutions of linear and nonlinear Fredholm IDEs with $\mathrm{AB}$ derivative.

Definition 10. The Laplace transform (LT) of $\mathcal{U}(t)$ is defined as [13]

$$
\mathbb{L}\{\mathcal{U}(t)\}=\widehat{\mathscr{U}}(s)=\int_{0}^{\infty} e^{-s t} \mathcal{U}(t) \mathrm{d} t .
$$

Definition 11. If $0<\alpha \leq 1$, then the $\mathrm{LT}$ of the $\mathrm{AB}$ derivative is defined by [52]

$$
\mathbb{L}\left\{{ }_{0}^{A B C} D_{t}^{\alpha} \mathscr{U}(t)\right\}=\frac{s^{\alpha} \widehat{\mathscr{U}}(s)-s^{\alpha-1} \mathcal{U}(0)}{s^{\alpha}(1-\alpha)+\alpha} .
$$

Applying the LT to equations (1) and(2), we obtain

$$
\frac{s^{\alpha} \widehat{\mathscr{U}}(s)-s^{\alpha-1} \mathscr{U}(0)}{s^{\alpha}(1-\alpha)+\alpha}=\hat{\mathscr{H}} \text {, }
$$

which can be rearranged as

$$
\widehat{\mathscr{U}}(s)=s^{-\alpha} \widehat{\mathscr{G}}(s),
$$

where

$$
\widehat{\mathscr{G}}(s)=s^{\alpha-1} \beta+\widehat{\mathscr{H}}\left(s^{\alpha}(1-\alpha)+\alpha\right) .
$$

The solution $\mathcal{U}(t)$ of equation (1) can be retrieved by writing it as a contour integral

$$
\mathcal{U}(t)=\frac{1}{2 \pi i} \int_{\Gamma} e^{\mathrm{st}} \hat{\mathcal{U}}(s) \mathrm{d} s, \quad \operatorname{Re}(s) \geq \sigma_{0},
$$

where $\sigma_{0} \in \mathbb{R}$ is large enough and the contour $\Gamma$ is a suitably selected line $\Gamma_{0}$ perpendicular to $x$-axis, with Ims $\longrightarrow \pm \infty$. Then, in (17), $\mathscr{U}(t)$ is the inverse Laplace of $\widehat{\mathcal{U}}(s)$, with the condition that the $\Gamma_{0}$ lies to the right of all the singularities of the transform $\hat{\mathscr{U}}(s)$. For our purposes, however, assuming that $\widehat{U}(s)$ may be continued analytically in an appropriate way, we shall want to take for $\Gamma_{0}$ a deformed contour $\Gamma$ in the set $\sum_{\phi}^{\delta}=\{0\} \cup\{s \neq 0$ : $|\arg s|<\phi\}$. The deformed contour will have asymptotic behavior as a couple of lines in the left complex plane, with Res $\longrightarrow-\infty$ and Ims $\longrightarrow \pm \infty$, which force the factor $e^{s t}$ to decay in the direction of both ends of the contour $\Gamma$. We define $\Gamma$ with the parametric representation of the form [54]

$$
s(\nu)=\delta+\xi-\xi \sin (\theta-\imath)), \quad \nu \in \mathbb{R},
$$

where

$$
0<\eta<\phi-\frac{\pi}{2}, \quad \xi>0, \text { and } \delta>0
$$

Letting $s=x+\imath y$, we notice that equation (18) serves as the left part of the hyperbola defined by

$$
\left(\frac{x-\delta-\xi}{\xi \sin \theta}\right)^{2}-\left(\frac{y}{\xi \cos \theta}\right)^{2}=1,
$$

where for equation (20), the asymptotes are $y= \pm(x-\delta-$ $\xi) \cot \theta$ and their $x$-intercept is $s=\delta+\xi(1-\sin \theta)$. Equation (19) ensures that the contour $\Gamma$ lies in $\Sigma_{\phi}^{\delta}=\delta+\Sigma_{\phi} \subset \Sigma_{\phi}$ and extends into the left complex plane. From (17) and (18), we obtain

$$
\mathcal{U}(t)=\frac{1}{2 \pi \iota} \int_{-\infty}^{\infty} e^{s(\nu) \tau} \widehat{\mathcal{U}}(s(\nu)) s^{\prime}(\nu) \mathrm{d} \nu
$$

The approximation of equation (21) can be obtained by employing the trapezoidal rule as

$$
\mathscr{U}_{k}(t)=\frac{k}{2 \pi \iota} \sum_{j=-N}^{N} e^{s_{j} t} \hat{\mathscr{U}}\left(s_{j}\right) s_{j}^{\prime}, \quad v_{j}=j k, s_{j}=s\left(v_{j}\right), s_{j}^{\prime}=s \prime\left(v_{j}\right) .
$$

2.1. Error Analysis of the Method. The process of approximating the solution of the problem defined in equations (1)-(3) involves the transformation of the given equation by employing the Laplace transform, and this process incurs no errors. We then obtain the solution of the transformed equation, and this process also incurs no errors. Finally, the solution of the problem is obtained via inverse Laplace transform by representing it as Bromwich integral (21). The integral is then approximated to high accuracy via trapezoidal rule. The process of approximating the integral (21) converges at different rates depending on the contour of integration $\Gamma$. Also, in this process, the convergence order is dependent on the step $k$ and on the chosen temporal domain $\left[t_{0}, T\right]$. For optimal results and best convergence, we chose an optimal temporal domain. The proof of the order quadrature error is provided in the following theorem.

Theorem 2 (see [54], Theorem 2.1). Let (1) has solution $\mathcal{U}(t)$ with $\hat{\mathcal{U}}(s)$ analytic in $\Sigma_{\phi}^{\delta}$. Let $\Gamma \subset \Omega_{r} \subset \Sigma_{\phi}^{\delta}$, and define $\cosh (b)=\left(\eta \tau_{1} \sin (\theta)\right)^{-1}, \quad$ for $\quad b>0$, where $0<\tau_{0}<T, \tau_{1}=\left(t_{0} / T\right), \quad 0<\eta<1$, and let $\beta=(\eta \bar{r} N / b T)$. Then, with $k=(b / N) \leq(\bar{r} / \log 2)$, for equation (22), we have $\left|\mathcal{U}(t)-\mathcal{U}_{k}(t)\right| \leq C Q e^{\delta \tau_{1}} l\left(\left\|\mathcal{U}_{0}\right\|+\|\hat{\mathscr{H}}\|_{\Sigma^{\delta}}\right)\left(\rho_{r} N\right) e^{-\mu N}, \quad$ for $\mu=(\bar{r}(1-\eta) t / b), \rho_{r}=\left(\eta \bar{r} \tau_{1} \sin \left(\eta-r_{1}\right) / b\right), \bar{r}=2 \pi r_{1}, r_{1}>0$, 
$\tau_{0} \leq t \leq T, l(x)=\max (1, \log (1 / x))$, and $C=C_{\eta, r_{1}, \beta_{1}}$. Hence, we have

$$
\text { error }_{\text {est }}=\left|\mathcal{U}_{k}(t)-\mathcal{U}(t)\right|=O\left(e^{-\mu N}\right) l\left(\rho_{r} N\right) \text {. }
$$

\section{Results and Discussion}

In this section, we consider linear and nonlinear Fredholmtype integrodifferential with $\mathrm{AB}$ derivative to validate our method. In our numerical experiments, the optimal values of the parameters $\delta=2.0, \eta=0.1, \bar{r}=2 \pi r$, $\theta=0.1541, \tau=(t 0 / T), r=0.1387$, and $\left[t_{0}, T\right]=[0.5,5.0]$ are used. We use the commands $\nu=-N: k: N$, for generating the nodes along the contour of integration.

3.1. Problem 1. Here, we consider linear Fredholm IDE with $\mathrm{AB}$ derivative of the form

$$
\left(\begin{array}{l}
{ }_{0}^{A B C} D_{t}^{\alpha} \\
0
\end{array}\right) \mathcal{U}(t)-\int_{0}^{1} t \tau \mathscr{U}(\tau) \mathrm{d} \tau=1-\frac{t}{3}, \quad t, \alpha \in[0,1] .
$$

The problem has exact solution $\mathcal{U}(t)=t$. Table 1 shows the numerical solution for various $\alpha$ and $t \in[0,1]$. It is observed that the results obtained for $\alpha=1$ are almost exact, whereas for other values of $\alpha$, the approximate solutions are in good agreement with the exact solution. Figure 1 displays the approximate solutions for different values of $\alpha$, while the absolute errors are shown in Figure 2 for $\alpha=1$. It is observed that the proposed method is an excellent alternative for solving such type of equations.

3.2. Problem 2. Here, a nonlinear Fredholm IDE is considered:

$$
\left(\begin{array}{ll}
{ }_{0}^{A B C} & D_{t}^{\alpha}
\end{array}\right) \mathcal{U}(t)-\int_{0}^{1} t \tau[\mathcal{U}(\tau)]^{2} \mathrm{~d} \tau=1-\frac{t}{4}, \quad t, \alpha \in[0,1] .
$$

The actual solution is $\mathcal{U}(t)=t$. The results for different $\alpha$ and various values of $N$ are depicted in Table 2 . Increase in accuracy of the method is observed with increase in the number of quadrature nodes. It is also observed the method produced exact solution for the fractional order $\alpha=1$ with large value of $N$, whereas the accuracy is decreased with decreasing the value of $\alpha$. The plots of approximate solutions for different $\alpha$ are displayed in Figure 3, while in Figure 4, for $\alpha=1$, the absolute error is presented.

3.3. Problem 3. Here, we consider the following nonlinear fractional Fredholm equation:

$$
\left(\begin{array}{l}
A B C \\
{ }_{0}^{A} D_{t}^{\alpha}
\end{array}\right) \mathcal{U}(t)-\int_{0}^{1} t^{2} \tau^{2}[\mathcal{U}(\tau)]^{2} \mathrm{~d} \tau=g(t), \quad t, \alpha \in[0,1]
$$

TABLE 1: The numerical results for various $\alpha$ and $N=210$.

\begin{tabular}{lcccc}
\hline$t$ & $\alpha=1$ & $\alpha=0.9$ & $\alpha=0.8$ & $\alpha=0.7$ \\
\hline 0 & 0 & 0.0583 & 0.1239 & 0.1964 \\
0.1 & 0.1 & 0.2312 & 0.3789 & 0.5406 \\
0.2 & 0.2 & 0.3395 & 0.4926 & 0.6550 \\
0.3 & 0.3 & 0.4422 & 0.5950 & 0.7526 \\
0.4 & 0.4 & 0.5415 & 0.6906 & 0.8408 \\
0.5 & 0.5 & 0.6384 & 0.7815 & 0.9225 \\
0.6 & 0.6 & 0.7333 & 0.8688 & 0.9994 \\
0.7 & 0.7 & 0.8266 & 0.9533 & 1.0726 \\
0.8 & 0.8 & 0.9186 & 1.0354 & 1.1426 \\
0.9 & 0.9 & 1.0095 & 1.1154 & 1.2101 \\
1 & 1 & 1.0993 & 1.1936 & 1.2754 \\
\hline
\end{tabular}

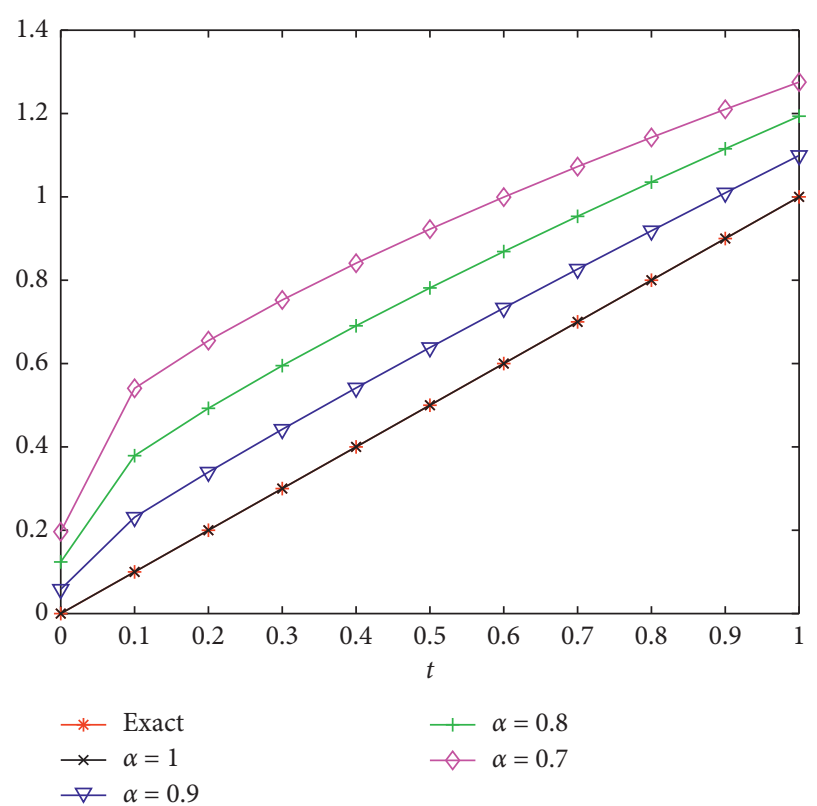

FIgURE 1: The plots of approximate solutions for fractional order $\alpha$ corresponding to problem 1 are shown. From this figure, it is clear that, for $\alpha=1$, the numerical and the exact solution are almost the same.

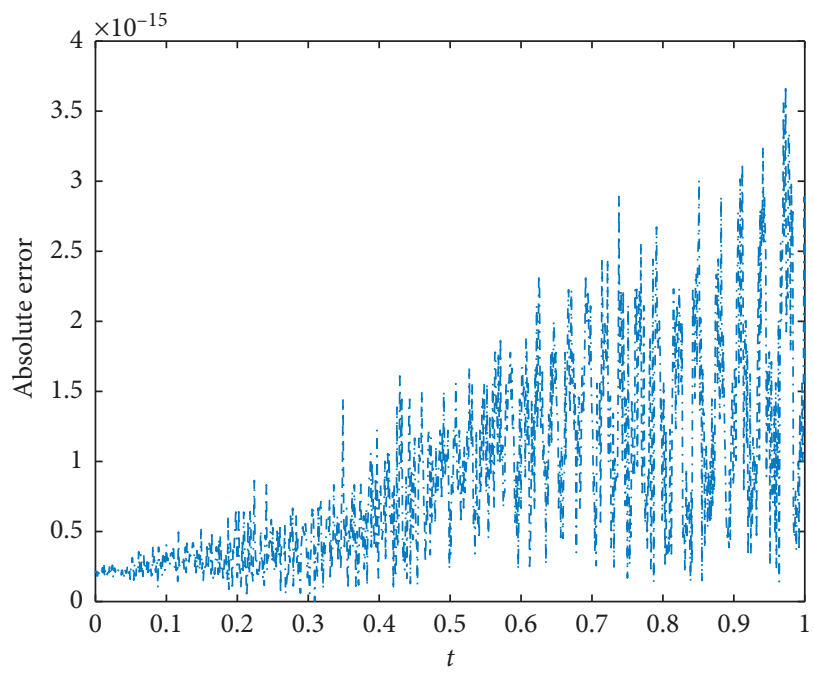

FIgURE 2: The plot of absolute error for $\alpha=1$ is shown. 
TABLE 2: Absolute errors for various quadrature points and different $\alpha$ at $t=1$.

\begin{tabular}{lcccc}
\hline$N$ & $\alpha=1$ & $\alpha=0.9$ & $\alpha=0.8$ & $\alpha=0.7$ \\
\hline 30 & $3.19 \times 10^{-4}$ & $3.83 \times 10^{-2}$ & $6.38 \times 10^{-2}$ & $7.76 \times 10^{-2}$ \\
50 & $1.51 \times 10^{-6}$ & $3.57 \times 10^{-2}$ & $5.87 \times 10^{-2}$ & $7.00 \times 10^{-2}$ \\
70 & $5.21 \times 10^{-7}$ & $3.58 \times 10^{-2}$ & $5.89 \times 10^{-2}$ & $7.04 \times 10^{-2}$ \\
90 & $4.25 \times 10^{-8}$ & $3.58 \times 10^{-2}$ & $5.89 \times 10^{-2}$ & $7.04 \times 10^{-2}$ \\
110 & $2.76 \times 10^{-9}$ & $3.58 \times 10^{-2}$ & $5.89 \times 10^{-2}$ & $7.04 \times 10^{-2}$ \\
130 & $1.58 \times 10^{-10}$ & $3.58 \times 10^{-2}$ & $5.89 \times 10^{-2}$ & $7.04 \times 10^{-2}$ \\
150 & $7.99 \times 10^{-12}$ & $3.58 \times 10^{-2}$ & $5.89 \times 10^{-2}$ & $7.04 \times 10^{-2}$ \\
170 & $3.37 \times 10^{-13}$ & $3.58 \times 10^{-2}$ & $5.89 \times 10^{-2}$ & $7.04 \times 10^{-2}$ \\
190 & $9.35 \times 10^{-15}$ & $3.58 \times 10^{-2}$ & $5.89 \times 10^{-2}$ & $7.04 \times 10^{-2}$ \\
210 & $3.99 \times 10^{-15}$ & $3.58 \times 10^{-2}$ & $5.89 \times 10^{-2}$ & $7.04 \times 10^{-2}$ \\
230 & $1.36 \times 10^{-15}$ & $3.58 \times 10^{-2}$ & $5.89 \times 10^{-2}$ & $7.04 \times 10^{-2}$ \\
\hline
\end{tabular}

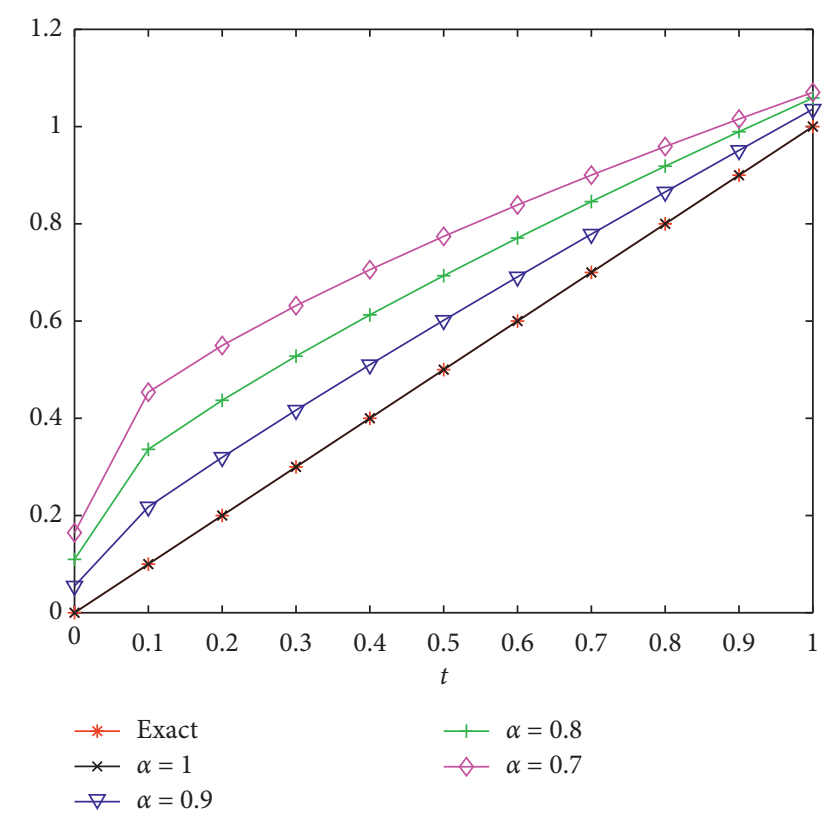

Figure 3: The plots of approximate solutions for fractional order $\alpha$ for problem 2 .

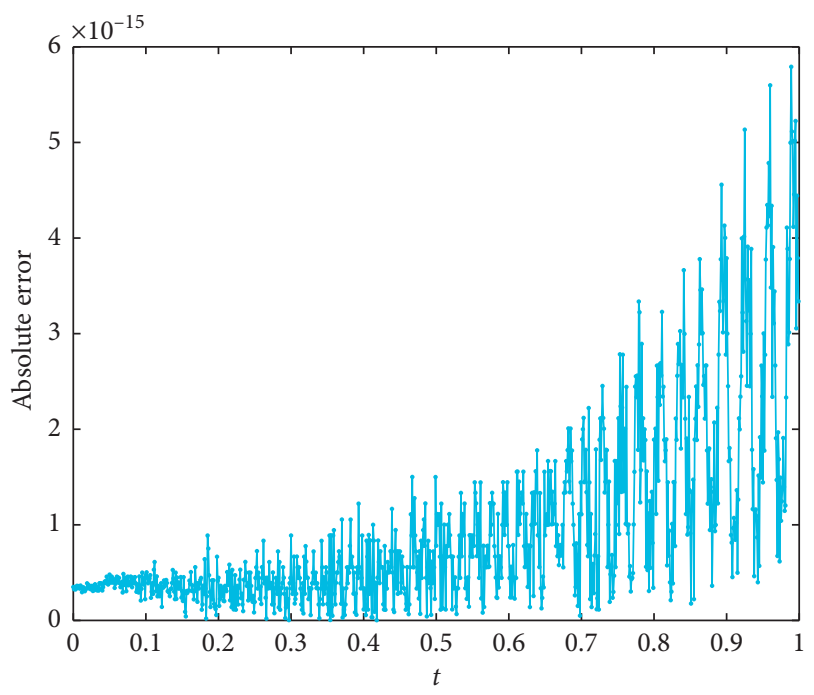

FIgURE 4: The plot of absolute error for $\alpha=1$.
TABle 3: The numerical results for various $\alpha$ and $N=250$.

\begin{tabular}{lcccc}
\hline$t$ & $\alpha=1$ & $\alpha=0.9$ & $\alpha=0.8$ & $\alpha=0.7$ \\
\hline 0.1 & 1.2000 & 1.4356 & 1.6723 & 1.9074 \\
0.2 & 1.4000 & 1.6397 & 1.8740 & 2.0994 \\
0.3 & 1.6000 & 1.8333 & 2.0557 & 2.2633 \\
0.4 & 1.8000 & 2.0205 & 2.2254 & 2.4113 \\
0.5 & 2.0000 & 2.2029 & 2.3867 & 2.5485 \\
0.6 & 2.2000 & 2.3818 & 2.5416 & 2.6776 \\
0.7 & 2.4000 & 2.5577 & 2.6914 & 2.8003 \\
0.8 & 2.6000 & 2.7310 & 2.8370 & 2.9180 \\
0.9 & 2.8000 & 2.9022 & 2.9790 & 3.0312 \\
1 & 3.0000 & 3.0716 & 3.1179 & 3.1408 \\
\hline
\end{tabular}

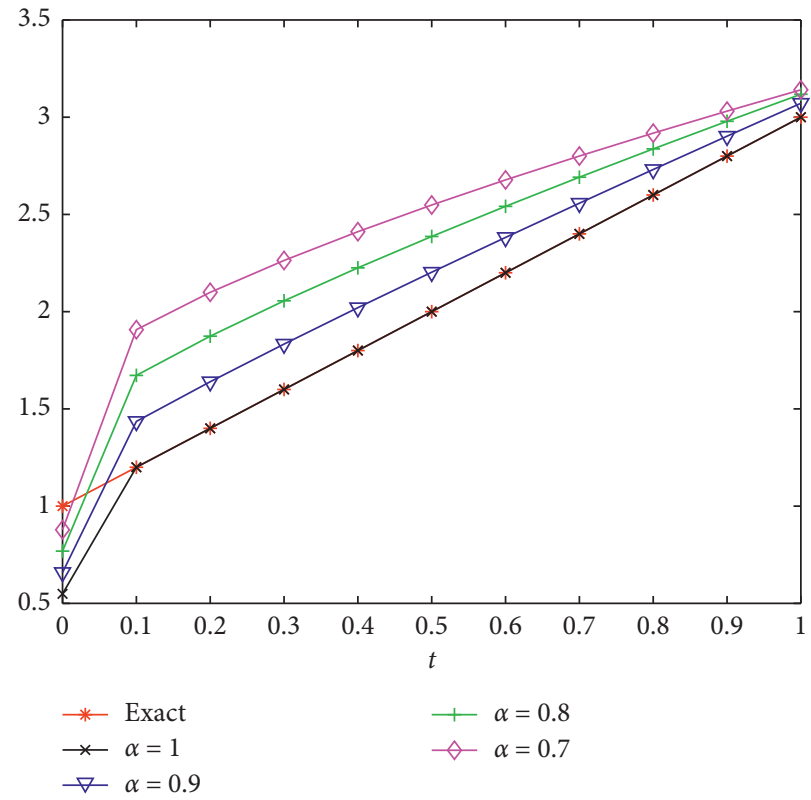

FIgURE 5: The plots of approximate solutions for fractional order $\alpha$ corresponding to problem 3 .

where

$$
g(t)=2-\frac{32}{15} t^{2}
$$

The problem has exact solution as $\mathcal{U}(t)=1+2 t$. The values of numerical solutions for different $\alpha$ and $t \in[0,1]$ are presented in Table 3. The results obtained for $\alpha=1$ are almost exact, whereas for other values of $\alpha$, the approximate solutions are in good agreement with the exact solution. The plots of approximate solutions for different $\alpha$ are presented in Figure 5, and the absolute errors are presented in Figure 6 for $\alpha=1$.

3.4. Problem 4. Let us consider a nonlinear Fredholm integrodifferential equation of the form

$$
\left(\begin{array}{l}
A B C \\
0
\end{array} D_{t}^{\alpha}\right) \mathcal{U}(t)-\int_{0}^{1} t \tau[\mathcal{U}(\tau)]^{2} \mathrm{~d} \tau=1-\frac{t^{3}}{3}, \quad t, \alpha \in[0,1] .
$$




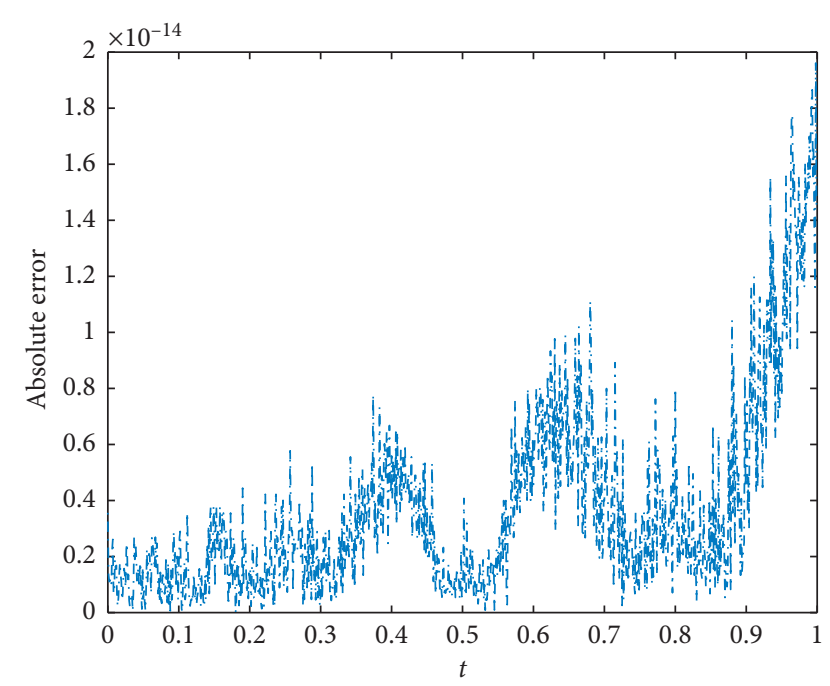

Figure 6: The plot of absolute error for $\alpha=1$.

TABLE 4: The numerical results for different $\alpha$ and various values of $N$.

\begin{tabular}{lccccc}
\hline$N$ & $\alpha=1$ & $\alpha=0.9$ & $\alpha=0.8$ & $\alpha=0.7$ & $\alpha=0.6$ \\
\hline 20 & $4.20 \times 10^{-3}$ & $9.89 \times 10^{-2}$ & $1.92 \times 10^{-1}$ & $2.72 \times 10^{-1}$ & $3.30 \times 10^{-1}$ \\
40 & $1.38 \times 10^{-4}$ & $9.92 \times 10^{-2}$ & $1.93 \times 10^{-1}$ & $2.75 \times 10^{-1}$ & $3.34 \times 10^{-1}$ \\
60 & $5.21 \times 10^{-6}$ & $9.93 \times 10^{-2}$ & $1.93 \times 10^{-1}$ & $2.75 \times 10^{-1}$ & $3.34 \times 10^{-1}$ \\
80 & $1.93 \times 10^{-7}$ & $9.93 \times 10^{-2}$ & $1.93 \times 10^{-1}$ & $2.75 \times 10^{-1}$ & $3.34 \times 10^{-1}$ \\
100 & $5.95 \times 10^{-9}$ & $9.93 \times 10^{-2}$ & $1.93 \times 10^{-1}$ & $2.75 \times 10^{-1}$ & $3.34 \times 10^{-1}$ \\
140 & $1.08 \times 10^{-11}$ & $9.93 \times 10^{-2}$ & $1.93 \times 10^{-1}$ & $2.75 \times 10^{-1}$ & $3.34 \times 10^{-1}$ \\
180 & $1.13 \times 10^{-13}$ & $9.93 \times 10^{-2}$ & $1.93 \times 10^{-1}$ & $2.75 \times 10^{-1}$ & $3.34 \times 10^{-1}$ \\
220 & $1.95 \times 10^{-16}$ & $9.93 \times 10^{-2}$ & $1.93 \times 10^{-1}$ & $2.75 \times 10^{-1}$ & $3.34 \times 10^{-1}$ \\
240 & $9.81 \times 10^{-16}$ & $9.93 \times 10^{-2}$ & $1.93 \times 10^{-1}$ & $2.75 \times 10^{-1}$ & $3.34 \times 10^{-1}$ \\
\hline
\end{tabular}

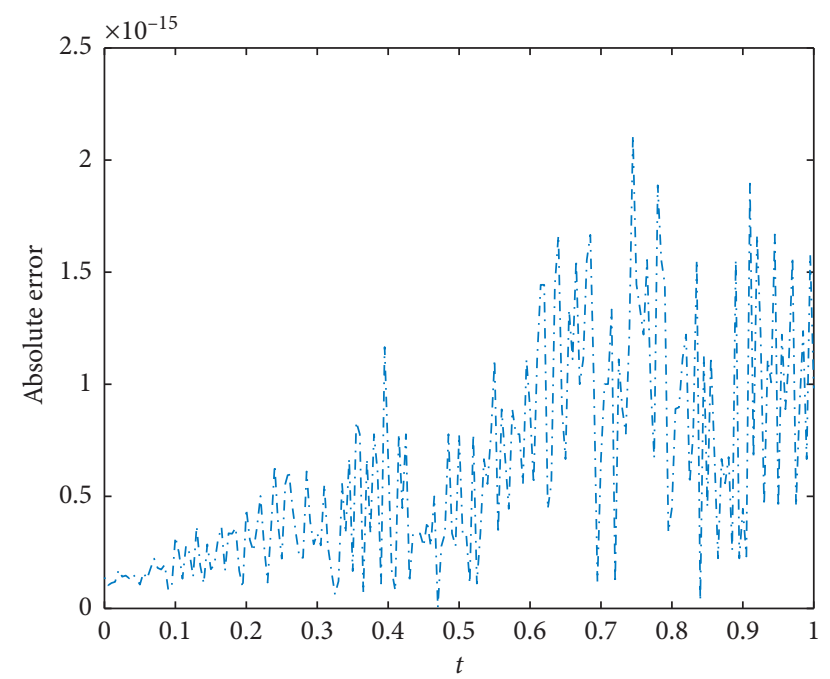

Figure 7: The absolute error for value $\alpha=1$ corresponding to problem 4 is shown.

The actual solution is $\mathcal{U}(t)=t$. The obtained results for $t \in[0,1]$ and different $\alpha$ are given in Table 4 . In Figure 7, the plot of absolute error for $\alpha=1$ is given, and in Figure 8, the graph of absolute error vs. error estimate is given.

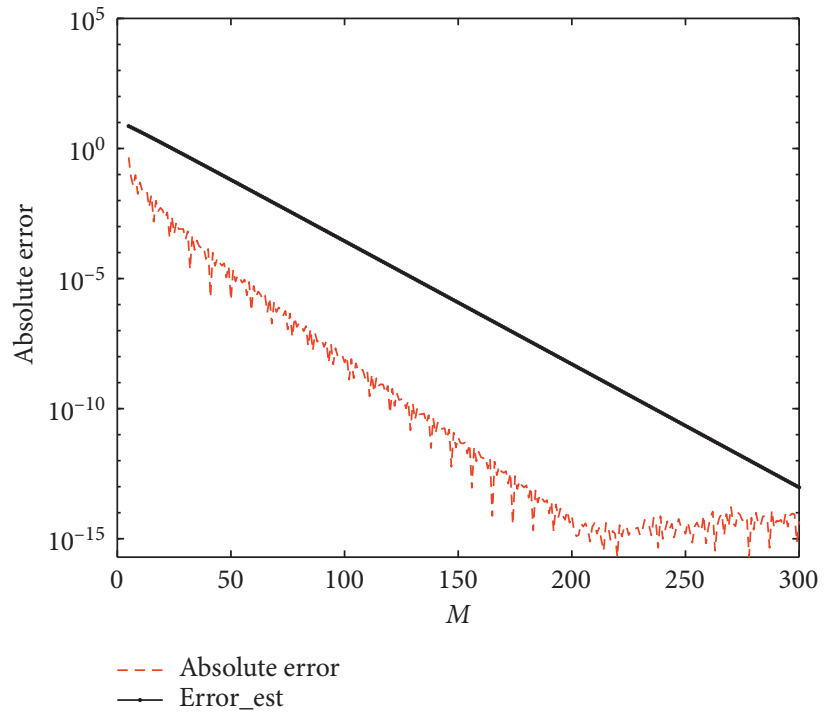

Figure 8: The absolute error and error estimate are shown for $\alpha=1$.

\section{Conclusion}

In this work, we solved the linear and nonlinear fractional Fredholm-type IDE with $A B$ derivative. We employed the LT to avoid the classical time-stepping method. The solution of the problem was obtained using inverse LT, by representing it as a contour integral along a smooth curve in the left half of complex plane. The contour integral was approximated using the trapezoidal rule. Error analysis and convergence of the method were discussed. The proposed scheme was applied to linear and nonlinear IDEs which produced accurate results. From the obtained results, it is observed that the proposed numerical scheme can solve such type of problems accurately. The main advantage of this method is that it gives us the solution at final time without any time instability issue, which is commonly encountered in time-stepping methods.

\section{Data Availability}

All data required for this research are included within the paper.

\section{Conflicts of Interest}

The authors declare that they do not have any conflicts of interest.

\section{Authors' Contributions}

Jian Wang proved the result, Kamran used the software to plot results and prepare tables, Ayesha Jamal revised the paper and improved the introduction, and Xuemei Li wrote the paper.

\section{References}

[1] C. Young, G. Farid, W. Nazeer, S. Ullah, and M. Shin, "Generalized riemann-liouville $k$-fractional integrals associated with ostrowski type inequalities and error bounds of 
hadamard inequalities," IEEE Access, vol. 6, pp. 64946-64953, 2018.

[2] S. Mehmood, G. Farid, and G. Farid, "Fractional integrals inequalities for exponentially $m$-convex functions," Open Journal of Mathematical Sciences, vol. 4, no. 1, pp. 78-85, 2020.

[3] G. Farid, "A unified integral operator and further its consequences," Open Journal of Mathematical Analysis, vol. 4, no. 1, pp. 1-7, 2020.

[4] S. Mehmood, G. Farid, G. Farid, K. A. Khan, and M. Yussouf, "New Hadamard and Fejér-Hadamard fractional inequalities for exponentially m-convex function," Engineering and Applied Science Letters, vol. 3, no. 1, p. 45, 2020.

[5] S. Mehmood, G. Farid, K. A. Khan, and M. Yussouf, "New fractional Hadamard and Fejér-Hadamard inequalities associated with exponentially $(h, m)$-convex functions," Engineering and Applied Science Letters, vol. 3, no. 9-18, 2020.

[6] R. L. Bagley and P. J. Torvik, "A theoretical basis for the application of fractional calculus to viscoelasticity," Journal of Rheology, vol. 27, pp. 201-210, 1983.

[7] J. Y. Hristov, "Linear viscoelastic responses: the prony decomposition naturally leads into the caputo-fabrizio fractional operator," Frontiers in Physics, vol. 6, no. 135, 2018.

[8] J. Hristov, "Transient heat diffusion with a non-singular fading memory: from the Cattaneo constitutive equation with Jeffrey's Kernel to the Caputo-Fabrizio time-fractional derivative," Thermal Science, vol. 20, no. 2, pp. 757-762, 2016.

[9] J. Hristov, "Derivation of the fractional dodson equation and beyond: transient diffusion with a non-singular memory and exponentially fading-out diffusivity," Progress in Fractional Differentiation and Applications, vol. 3, no. 4, pp. 255-270, 2017.

[10] R. T. Baillie, "Long memory processes and fractional integration in econometrics," Journal of Econometrics, vol. 73, no. 5-59, 1996.

[11] G. W. Bohannan, "Analog fractional order controller in temperature and motor control applications," Journal of Vibration and Control, vol. 14, no. 9-10, pp. 1487-1498, 2008.

[12] L. Debnath, "Recent applications of fractional calculus to science and engineering," International Journal of Mathematics and Mathematical Sciences, vol. 2003, no. 54, pp. 3413-3442, 2003.

[13] I. Podlubny, Fractional Differential Equations: An Introduction to Fractional Derivatives, Fractional Differential Equations, to Methods of Their Solution and Some of Their Applications, Elsevier, Amsterdam, Netherlands, 1998.

[14] A. Arikoglu and I. Ozkol, "Solution of fractional integrodifferential equations by using fractional differential transform method," Chaos, Solitons \& Fractals, vol. 40, no. 2, pp. 521-529, 2009.

[15] O. A. Arqub and B. Maayah, "Numerical solutions of integrodifferential equations of Fredholm operator type in the sense of the Atangana-Baleanu fractional operator," Chaos, Solitons \& Fractals, vol. 117, pp. 117-124, 2018.

[16] A. A. Kilbas, H. M. Srivastava, and J. J. Trujillo, Theory And Applications Of Fractional Differential Equations, Vol. 204, Elsevier Science Limited, Amsterdam, Netherlands, 2006.

[17] M. M. A. Khater, R. A. M. Attia, and A. Abdel-Aty, "Computational analysis of a nonlinear fractional emerging telecommunication model with higher-order dispersive cubic-quintic," Information Sciences Letters, vol. 9, no. 2, 2020.

[18] G. M. Ismail, H. R. Abdl-Rahim, A. Abdel-Aty, R. Kharabsheh, W. Alharbi, and M. Abdel-Aty, "An analytical solution for fractional oscillator in a resisting medium," Chaos, Solitons \& Fractals, vol. 130, p. 109395, 2020.

[19] S. O. Edeki, I. Adinya, G. O. Akinlabi, and O. P. Ogundile, "Conformable decomposition for analytical solutions of a time-fractional one-factor markovian model for bond pricing," Applied Mathematics \& Information Sciences, vol. 13, no. 4, pp. 539-544, 2019.

[20] H. Khan, A. Khan, F. Jarad, and A. Shah, "Existence and data dependence theorems for solutions of an abc-fractional order impulsive system," Chaos, Solitons \& Fractals, vol. 131, p. $109477,2020$.

[21] A. Saadatmandi and M. Dehghan, "A legendre collocation method for fractional integro-differential equations," Journal of Vibration and Control, vol. 17, no. 13, pp. 2050-2058, 2011.

[22] S. Alkan and V. F. Hatipoglu, "Approximate solutions of Volterra-Fredholm integro-differential equations of fractional order," Tbilisi Mathematical Journal, vol. 10, no. 2, pp. 1-13, 2017.

[23] Y. Nawaz, "Variational iteration method and homotopy perturbation method for fourth-order fractional integrodifferential equations," Computers \& Mathematics with Applications, vol. 61, no. 8, pp. 2330-2341, 2011.

[24] S. Momani and M. Aslam Noor, "Numerical methods for fourth-order fractional integro-differential equations," $A p$ plied Mathematics and Computation, vol. 182, no. 1, pp. 754-760, 2006.

[25] S. Owyed, M. A. Abdou, A. H. Abdel-Aty, W. Alharbi, and R. Nekhili, "Numerical and approximate solutions for coupled time fractional nonlinear evolutions equations via reduced differential transform method," Chaos, Solitons \& Fractals, vol. 131, p. 109474, 2020.

[26] C. M. D. Simarmata, N. Susyanto, I. J. Hammadi, and C. Rahmaditya, "A mathematical model of smoking behaviour in Indonesia with density-dependent death rate," Open Journal of Mathematical Sciences, vol. 4, no. 1, pp. 118-125, 2020.

[27] E. Ul Haq, M. Ali, and A. S. Khan, "On the solution of fractional riccati differential equations with variation of parameters method," Engineering and Applied Science Letter, vol. 3, no. 3, pp. 1-9, 2020.

[28] H. Ahmed, B. Hamid, and A. Ardjouni, "Existence and uniqueness for delay fractional differential equations with mixed fractional derivatives," Open Journal of Mathematical Anaysis, vol. 4, no. 2, pp. 26-31, 2020.

[29] K. Issa, K. M. Humbali, S. M. Hambali, and J. Biazar, “An algorithm for choosing best shape parameter for numerical solution of partial differential equation via inverse multiquadric radial basis function," Open Journal of Mathematical Sciences, vol. 4, no. 1, pp. 147-157, 2020.

[30] A. T. Ali, M. M. A. Khater, R. A. M. Attia, A. H. Abdel-Aty, and D. Lu, "Abundant numerical and analytical solutions of the generalized formula of Hirota-Satsuma coupled $\mathrm{KdV}$ system," Chaos, Solitons \& Fractals, vol. 131, p. 109473, 2020.

[31] M. Y. Abubakar, L. T. Jung, N. Zakaria, A. Younes, and A.-H. Abdel-Aty, "Reversible circuit synthesis by genetic programming using dynamic gate libraries," Quantum Information Processing, vol. 16, no. 6, p. 160, 2017.

[32] A. R. Hadhoud, "Quintic non-polynomial spline method for solving the time fractional biharmonic equation," Applied Mathematics \& Information Sciences, vol. 13, no. 3, pp. 507513, 2019.

[33] V. E. Tarasov, "Fractional integro-differential equations for electromagnetic waves in dielectric media," Theoretical and Mathematical Physics, vol. 158, no. 3, pp. 355-359, 2009. 
[34] R. P. Agarwal, B. de Andrade, and G. Siracusa, "On fractional integro-differential equations with state-dependent delay," Computers \& Mathematics with Applications, vol. 62, no. 3, pp. 1143-1149, 2011.

[35] E. A. Rawashdeh, "Numerical solution of fractional integrodifferential equations by collocation method," Applied Mathematics and Computation, vol. 176, no. 1, pp. 1-6, 2006.

[36] E. A. Rawashdeh, "Legendre wavelets method for fractional integro-differential equations," Applied Mathematical Sciences, vol. 5, no. 2, pp. 2467-2474, 2011.

[37] S. G. Samko, A. A. Kilbas, and O. I. Marichev, Fractional Integrals And Derivatives, Vol. 1, Gordon and Breach Science Publishers, Yverdon Yverdon-les-Bains, Switzerland, 1993.

[38] M. A. Abdelkawy and I. G. Ameen, "A spectral collocation method for coupled system of two dimensional abel integral equations of the second kind," Information Sciences Letters, vol. 8, pp. 89-93, 2019.

[39] X. Ma and C. Huang, "Numerical solution of fractional integro-differential equations by a hybrid collocation method," Applied Mathematics and Computation, vol. 219, no. 12, pp. 6750-6760, 2013.

[40] S. Mashayekhi and M. Razzaghi, "Numerical solution of nonlinear fractional integro-differential equations by hybrid functions," Engineering Analysis with Boundary Elements, vol. 56, pp. 81-89, 2015.

[41] A. Khan, Y. Li, K. Shah, and T. S. Khan, "On coupled plaplacian fractional differential equations with nonlinear boundary conditions," Complexity, vol. 2017, Article ID 8197610, 9 pages, 2017.

[42] A. Khan, T. Abdeljawad, J. F. Gómez-Aguilar, and H. Khan, "Dynamical study of fractional order mutualism parasitism food web module," Chaos, Solitons \& Fractals, vol. 134, p. 109685, 2020.

[43] M. Shin, G. Farid, W. Nazeer, and S. Mehmood, $(h-m)$ "-convex functions and associated fractional hadamard and Fejér-Hadamard inequalities via an extended generalized Mittag-Leffler function," Journal of Inequalities and Applications, vol. 2019, no. 1, pp. 1-10, 2019.

[44] M. Shin, G. Farid, W. Nazeer, and B. Tariq, "Hadamard and Fejér-Hadamard inequalities for extended generalized fractional integrals involving special functions," Journal of Inequalities and Applications, vol. 2018, no. 1, p. 119, 2018.

[45] C. Young, G. Farid, S. Ullah, W. Nazeer, K. Mahreen, and M. Shin, "Inequalities for a unified integral operator and associated results in fractional calculus," IEEE Access, vol. 7, pp. 126283-126292, 2019.

[46] X. Yang, G. Farid, G. Farid, W. Nazeer, Y.-M. Chu, and C. Dong, "Fractional generalized Hadamard and FejérHadamard inequalities for," AIMS Math, vol. 5, no. 6, pp. 6325-6340, 2020.

[47] S. Zhao, S. I. Butt, W. Nazeer, J. Nasir, M. Umar, and Y. Liu, "Some Hermite-Jensen-mercer type inequalities for $k$ caputo-fractional derivatives and related results," Advances in Difference Equations, vol. 2020, no. 1, pp. 1-17, 2020.

[48] A. Atangana and J. F. Gómez-Aguilar, "Decolonisation of fractional calculus rules: breaking commutativity and associativity to capture more natural phenomena," The European Physical Journal Plus, vol. 133, no. 4, p. 166, 2018.

[49] M. A. Abdou, S. Owyed, A. Abdel-Aty, B. M. Raffah, and S. Abdel-Khalek, "Optical soliton solutions for a space-time fractional perturbed nonlinear Schrödinger equation arising in quantum physics," Results in Physics, vol. 16, p. 102895, 2020 .
[50] H. Khan, J. F. Gómez-Aguilar, A. Alkhazzan, and A. Khan, “A fractional order HIV-TB coinfection model with nonsingular Mittag-Leffler law," Mathematical Methods in the Applied Sciences, vol. 43, no. 6, pp. 3786-3806, 2020.

[51] X. Liu, Kamran, and Y. Yao, "Numerical approximation of Riccati fractional differential equation in the sense of Caputotype fractional derivative," Journal of Mathematics, vol. 2020, Article ID 1274251, 12 pages, 2020.

[52] A. Atangana and D. Baleanu, "New fractional derivatives with nonlocal and non-singular kernel: theory and application to heat transfer model," Thermal Science, vol. 20, pp. 763-769, 2016.

[53] C. Ravichandran, K. Logeswari, and F. Jarad, "New results on existence in the framework of Atangana-Baleanu derivative for fractional integro-differential equations," Chaos, Solitons \& Fractals, vol. 125, pp. 194-200, 2019.

[54] W. McLean and V. Thomee, "Numerical solution via Laplace transforms of a fractional order evolution equation," Journal of Integral Equations and Applications, vol. 22, no. 1, pp. 57-94, 2010. 